\title{
Age-related cognitive decline is indicative of neuropathology
}

Rachel Buckley, $\mathrm{PhD}^{1,2,3,4}$; Alvaro Pascual-Leone, MD, $\mathrm{PhD}^{4,5,6}$

\author{
${ }^{1}$ Department of Neurology, Massachusetts General Hospital, Boston, MA, USA \\ ${ }^{2}$ Center for Alzheimer Research and Treatment, Department of Neurology, Brigham and \\ Women's Hospital, Boston, Massachusetts, USA \\ ${ }^{3}$ Melbourne School of Psychological Science, University of Melbourne, Victoria, Australia \\ ${ }^{4}$ Department of Neurology, Harvard Medical School, Boston, MA, USA \\ ${ }^{5} \mathrm{Hinda}$ and Arthur Marcus Institute for Aging Research and Center for Memory Health, Hebrew \\ SeniorLife, Boston, MA, USA \\ ${ }^{6}$ Guttmann Brain Health Institute, Institut Guttmann de Neurorehabilitació, Universitat \\ Autónoma, Barcelona, Spain
}

The extension of the human lifespan has not translated into a parallel extension of a healthy lifespan (or healthspan). Cognitive impairment and dementia are the most common diagnoses that impact the healthspan as we age. However, a debate remains as to whether cognitive decline may reflect simply aging-related changes in brain function, often referred to as 'normative aging', or whether it indicates the presence of neuropathology, 'non-normative processes'1.

Aging is associated with an increased risk in a large number of debilitating conditions, including neurologic and psychiatric diseases. 'Non-normative' processes represent factors associated with general health and medical conditions (vascular factors, atherosclerosis, infarcts, stroke), biological processes (disease-related pathology, inflammation) and genetics. Any residual amount of cognitive slowing not explained by such 'non-normative' processes is assumed to represent the effect of senescence (normative aging) ${ }^{1}$.

A broadly made assumption is that both normative and non-normative processes meaningfully contribute to an individuals' cognitive trajectory across the lifespan. However, a large body of epidemiologic data following cognitive trajectories of well-characterized adults suggests that the very notion of normative cognitive decline can be challenged. Going back to pioneering work by Park et al. $^{2}$ it seems clear that some cognitive abilities worsen with increasing age. These include for example processing speed, working memory and long-term memory. However, other cognitive abilities improve with age, notably those linked to crystallized knowledge and vocabulary. Thus, some individuals seem to sustain overall cognitive capacity despite advancing age and are often referred to as 'maintainers', whilst others show an agerelated cognitive decline and are referred to as 'decliners' 3 (Figure 1). A predominant tenet is that decliners reflect the consequences of normative aging while maintainers benefit from particular brain capacities of resilience or reserve enabling them to sustain cognitive function despite the aging process ${ }^{4}$. In this issue of Annals of Neurology, Wilson et al. ${ }^{5}$ directly test these notions and provide extremely important and novel insights.

Wilson et al. ${ }^{5}$ examined the impact of age-at-death and neuropathology on cognitive decline trajectories in individuals from the Religious Orders Study and Rush Memory and Aging

This is the author manuscript accepted for publication and has undergone full peer review but has not been through the copyediting, typesetting, pagination and proofreading process, which may lead to differences between this version and the Version of Record. Please cite this article as doi: $10.1002 /$ ana. 25733

This article is protected by copyright. All rights reserved. 
Project (ROSMAP) cohorts. After adjusting for major neuropathological factors (Alzheimer's disease [AD], TDP-43, neocortical Lewy bodies [LBD], hippocampal sclerosis, cerebral amyloid angiopathy [CAA], gross/microinfarct, atherosclerosis and arteriosclerosis) age at death remained only modestly associated with rate of global cognitive decline. Thus, it seems that neuropathology, i.e. non-normative factors, account for the vast majority of age-related cognitive decline.

Wilson et al. ${ }^{5}$ used a statistical approach, functional mixed effects, that allowed for individuals to vary flexibly and non-linearly over time, leading to a more robust estimation of the effects on cognitive trajectories. They determined that many neuropathologic factors impacted negatively on global cognitive function over time, and that each factor exerted a different nonlinear/linear association to predict decline. For instance, hippocampal sclerosis associated with a linear trend of cognitive decline prior to death, while neuropathologies such as AD and neocortical LBD exerted a curvilinear influence of accelerated decline. Purely age-related (i.e. 'normative') processes had only a weak effect on global cognitive trajectories, which was driven by age-associated changes in perceptual speed but not episodic memory. Further, after adjusting for non-normative factors, a large variability in residual cognitive trajectories was apparent. Upon clustering these longitudinal residuals, only a subgroup of individuals was found to exhibit precipitous cognitive change prior to death which presumably is due to a milieu of mortality-related influences ${ }^{6}$. Therefore, the construct of "normative aging" cannot explain declining cognitive trajectories. Instead, a majority of decline trajectories can be almost entirely explained by non-normative processes - notably by underlying brain pathology and the individual's inability to cope with it.

A noteworthy aspect of these findings is the non-linearity apparent in the associations between variables such as AD/LBD neuropathology and cognition. Although curvilinear patterns in cognitive trajectories have been observed in a range of cohorts ${ }^{7-10}$, particularly as individuals progress to a clinical diagnosis, the prevailing approach to modelling involves an assumption of linearity. If we assume linearity, the inflection points at which cognitive decline accelerates with encroaching disease could be poorly estimated, or worse, not modelled at all. It is clear from Wilson et al. ${ }^{5}$ that we should consider employing non-linear approaches to better approximate the cognitive point cloud. Techniques, such as functional mixed effects models using B-spline fit, ${ }^{5}$ random change-point models ${ }^{9},{ }^{10}$, or mixed effect models of repeated measures (MMRM) ${ }^{7}$ should be favored over more commonly used linear mixed effects models.

Wilson and colleagues' findings also have implications for the concept of cognitive reserve, particularly with regard to the maintenance of cognitive performance for given a level of neuropathological burden. Wilson et al. ${ }^{5}$ found an appreciable number of individuals maintain or even increase - their cognitive capacity above and beyond their level of disease burden. It is as yet unclear what lifestyle factors and neurobiological mechanisms may explain this maintenance or improvement of cognitive reserve over time ${ }^{11-13}$. It is important to consider genome-wide associations ${ }^{14}$, but also life-long modifiable lifestyles in different domains including nutrition, sleep, physical exercise, cognitive stimulation, and particularly social interactions and a well-defined purpose in life, that seem to play critical roles and offer targets for intervention ${ }^{15-18}$. 
Finally, the findings by Wilson et al. ${ }^{5}$ have practical implications. Age-adjusted cognitive data is used to define impairment in the clinic. Estimates from current norms likely include data from individuals in the same age range, some of whom harbor clinically unobservable levels of 'non-normative' conditions (e.g. cardiovascular disease or AD) making these norms much more difficult to interpret. This approach is particularly limiting for the oldest age ranges where the overall number of individuals is less and those without some underlying pathology may be very few. Wilson and colleagues suggest a more appropriate approach may be to compare the performance of older individuals to those who are much more likely to be relatively unencumbered from non-normative and mortality factors. Complementary approaches may consider incorporating metrics of brain function that might identify individuals at risk for cognitive decline years prior to the manifestation of impairment in neuropsychological task performance. Ultimately, frequent, longitudinal assessments are essential to detect deviations of an individual's cognitive trajectory that may herald the presence of neuropathology.

The current study joins an increasing body of evidence that suggests age-associated cognitive decline may be an explanation of the past. With increasingly more sophisticated approaches to modelling the influence of neuropathological burden on longitudinal cognitive trajectories in studies like ROSMAP, it is hard to justify the notion that age represents a 'driver' of decline. From these findings using post-mortem neuropathological outcome data, it is apparent that unexplained cognitive variance in in vivo models of disease may not be representing "normative" aging processes, but an as-yet unmeasured non-normative processes (Figure 1). As our in vivo measures improve (e.g. blood and CSF analytes, novel PET tracers, perturbation-biomarkers combining noninvasive brain stimulation with brain imaging or EEG, etc.) we will be able to run increasingly powerful models of change in neuropathological burden with cognition to delve into the likely factors that impact non-linear trajectories.

It seems that the implementation of appropriately sensitive brain health and cognitive function evaluations as part of yearly check-ups is thus overdue. To fully realize this goal, we need more sensitive screening methods to identify meaningful changes in individual cognitive trajectories that may herald brain pathological processes. By the time patients become symptomatic and a diagnosis is made, the impact of brain diseases is already significant and complex, and intricate processes of adaptation - both of brain structure and function, and of individual and social behavior - have become established. At that point, therapeutic interventions can rarely achieve a cure and have limited impact on disability. Digital technology provides a unique opportunity to collect lifestyle, health history, genetic information, and large amounts of real-time, ecologically valid metrics of cognitive function and behavior. This will enable machine learning models to detect specific dementia-causing diseases many years before cognitive symptoms. Ultimately, earlier diagnosis will allow more timely interventions to prevent disability.

\section{References}


1. Deary IJ, Corley J, Gow AJ, et al. Age-associated cognitive decline. British medical bulletin. 2009;92(1):135-52.

2. Park DC, Reuter-Lorenz $P$. The adaptive brain: aging and neurocognitive scaffolding. Annu Rev Psychol. 2009;60:173-96.

3. Yaffe $\mathrm{K}$, Fiocco AJ, Lindquist $\mathrm{K}$, et al. Predictors of maintaining cognitive function in older adults: the Health ABC study. Neurology. 2009;72(23):2029-35.

4. Stern Y, Arenaza-Urquijo EM, Bartrés-Faz D, et al. Whitepaper: Defining and investigating cognitive reserve, brain reserve, and brain maintenance. Alzheimer's \& Dementia. 2018.

5. Wilson R, Wang T, Yu L, Bennett D, Boyle P. Normative Cognitive Decline in Old Age. Annals of Neurology. 2020;xx(xx):xx.

6. Wilson RS, Yu L, Leurgans SE, Bennett DA, Boyle PA. Proportion of cognitive loss attributable to terminal decline. Neurology. 2020;94(1):e42-e50.

7. Donohue MC, Sperling RA, Petersen R, Sun C-K, Weiner MW, Aisen PS. Association Between Elevated Brain Amyloid and Subsequent Cognitive Decline Among Cognitively Normal Persons. JAMA. 2017;317(22):2305-16.

8. Buckley RF, Mormino EC, Amariglio RE, et al. Sex, Amyloid, and APOEe4 and risk of cognitive decline in preclinical Alzheimer's disease: findings from three well-characterized cohorts. Alzheimer's \& Dementia. 2018;14(9):1193-203.

9. Jansen WJ, Wilson RS, Visser PJ, et al. Age and the association of dementia-related pathology with trajectories of cognitive decline. Neurobiology of aging. 2018;61:138-45.

10. Williams OA, An Y, Armstrong NM, Kitner-Triolo M, Ferrucci L, Resnick SM. Profiles of cognitive change in preclinical Alzheimer's disease using change-point analysis. medRxiv. 2019:19009696.

11. Zahodne LB, Manly JJ, Brickman AM, Siedlecki KL, DeCarli C, Stern Y. Quantifying cognitive reserve in older adults by decomposing episodic memory variance: replication and extension. Journal of the International Neuropsychological Society. 2013;19(8):85462.

12. Zahodne LB, Manly JJ, Brickman AM, et al. Is residual memory variance a valid method for quantifying cognitive reserve? A longitudinal application. Neuropsychologia. 2015;77:260-6.

13. Bettcher BM, Gross AL, Gavett BE, et al. Dynamic change of cognitive reserve: associations with changes in brain, cognition, and diagnosis. Neurobiology of aging. 2019;83:95-104.

14. Hohman TJ, Dumitrescu L, Cox NJ, Jefferson AL. Genetic resilience to amyloid related cognitive decline. Brain Imaging and Behavior. 2017;11(2):401-9. 
15. Baumgart M, Snyder HM, Carrillo MC, Fazio S, Kim H, Johns H. Summary of the evidence on modifiable risk factors for cognitive decline and dementia: a populationbased perspective. Alzheimer's \& Dementia. 2015;11(6):718-26.

16. Kivipelto $\mathrm{M}$, Mangialasche $\mathrm{F}, \mathrm{Ngandu} \mathrm{T}$. Lifestyle interventions to prevent cognitive impairment, dementia and Alzheimer disease. Nature Reviews Neurology. 2018;14(11):653-66.

17. Cattaneo G, Bartrés-Faz D, Morris TP, et al. The barcelona brain health initiative: a cohort study to define and promote determinants of brain health. Frontiers in aging neuroscience. 2018;10:321.

18. Laurino M, Alfì G, Billeci L, et al. Healthy aging: the INTECMAN project. Aging Clinical and Experimental Research. 2020:1-5.

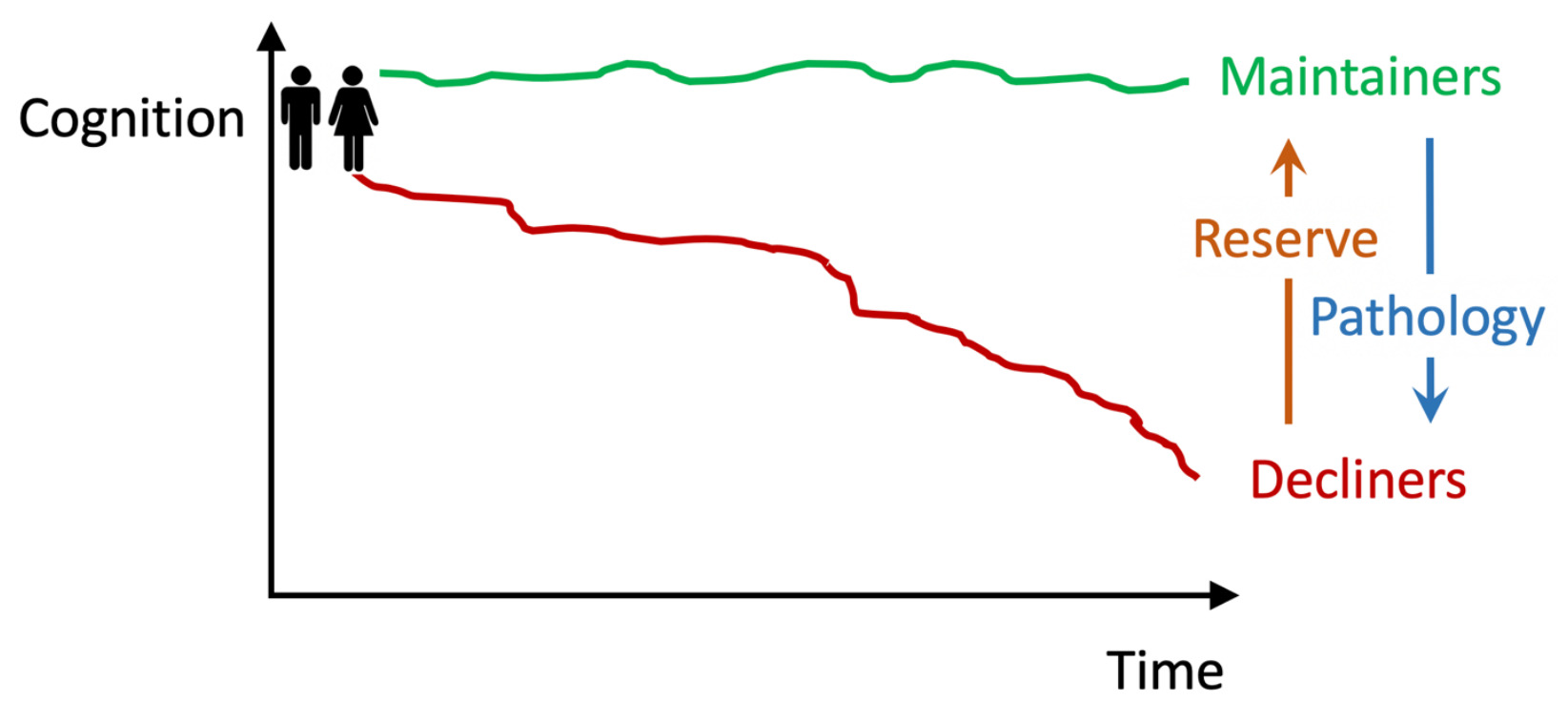

Figure 1. Diagrammatic representation of the influence of neuropathological burden on cognitive performance over time, which can result in maintainer and decliner trajectories. 


\section{University Library}

\section{- M M I N E R VA A gateway to Melbourne's research publications}

Minerva Access is the Institutional Repository of The University of Melbourne

Author/s:

Buckley, R;Pascual-Leone, A

Title:

Age-Related Cognitive Decline Is Indicative of Neuropathology

Date:

2020-04-24

Citation:

Buckley, R. \& Pascual-Leone, A. (2020). Age-Related Cognitive Decline Is Indicative of Neuropathology. ANNALS OF NEUROLOGY, 87 (6), pp.813-815. https://doi.org/10.1002/ ana.25733.

Persistent Link:

http://hdl.handle.net/11343/275681 